Article

\title{
Activation Volume and Energy for Dislocation Nucleation in Multi-Principal Element Alloys
}

\author{
Sanghita Mridha, Maryam Sadeghilaridjani and Sundeep Mukherjee *(1) \\ Department of Materials Science and Engineering, University of North Texas, Denton, TX 76203, USA; \\ sanghitamridha@my.unt.edu (S.M.); Maryam.Sadeghilaridjani@unt.edu (M.S.) \\ * Correspondence: sundeep.mukherjee@unt.edu; Tel.: +1-940-565-4170; Fax: +1-940-565-2944
}

Received: 25 January 2019; Accepted: 21 February 2019; Published: 23 February 2019

\begin{abstract}
Incipient plasticity in multi-principal element alloys, $\mathrm{CoCrNi}, \mathrm{CoCrFeMnNi}$, and $\mathrm{Al}_{0.1} \mathrm{CoCrFeNi}$ was evaluated by nano-indentation and compared with pure Ni. The tests were performed at a loading rate of $70 \mu \mathrm{N} / \mathrm{s}$ in the temperature range of $298 \mathrm{~K}$ to $473 \mathrm{~K}$. The activation energy and activation volume were determined using a statistical approach of analyzing the "pop-in" load marking incipient plasticity. The $\mathrm{CoCrFeMnNi}$ and $\mathrm{Al}_{0.1} \mathrm{CoCrFeNi}$ multi-principal element alloys showed two times higher activation volume and energy compared to $\mathrm{CoCrNi}$ and pure $\mathrm{Ni}$, suggesting complex cooperative motion of atoms for deformation in the five component systems. The small calculated values of activation energy and activation volume indicate heterogeneous dislocation nucleation at point defects like vacancy and hot-spot.
\end{abstract}

Keywords: multi-principal element alloys; dislocation nucleation; activation volume; activation energy; nano-indentation; high/medium entropy alloys

\section{Introduction}

Traditional alloy design employs the addition of alloying elements to a principal constituent to enhance its properties via multi-phase complex microstructure. Multi-principal element alloys (MPEAs) represent a new generation of material system consisting of several elements in equimolar or near equimolar proportions. Despite the complex chemistry, high configurational entropy may favor formation of a simple solid solution instead of complex intermetallic compounds in these alloys, which are also referred to as high entropy alloys (HEAs) [1]. Certain range in atomic size difference, electronegativity difference, and mixing entropy/enthalpy of the constituent elements result in multiple concentrated phases in the microstructure [2-5].

The mechanical properties of MPEAs have been widely studied in recent years over a range of temperatures for potential applications in nuclear and aerospace industries. These include tensile/compressive properties, fatigue behavior, and fracture toughness [6-12]. It has been hypothesized that MPEAs have a distorted lattice structure which makes dislocation movement difficult and non-traditional in these alloy systems [1,6-8,13-18]. To understand dislocation nucleation and the activation process, nano-indentation technique has been widely used. The displacement burst (pop-in) in the nano-indentation load versus contact depth curve is attributed to dislocation nucleation. The "pop-in" represents the transition from elastic to plastic deformation in materials and typically happens at a small indentation depth of $<100 \mathrm{~nm}$ [19-26]. The stress associated with the pop-in is approximately equal to the theoretical strength of the material and happens in small indented volume with a very low probability of having pre-existing dislocations. Therefore, many researchers have attributed the pop-in event to dislocation nucleation [21,25,27-33], and others have proposed vacancy assisted mechanisms [34]. It has been demonstrated that the pop-in during nano-indentation is time and temperature dependent, a kinetically limiting process that requires activation energy. It occurs in 
thermally and mechanically favorable positions, which makes it a probabilistic event, i.e., the load at which the pop-in takes place is distributed over a range rather than occurring at a fixed value each time, and this distribution is temperature and time dependent.

Nano-indentation technique has been extensively used to study the local scale elastic-plastic deformation behavior of metals/alloys and to determine the critical shear stress required for dislocation nucleation [19-26]. However, there are few reports and limited understanding of the effect of intrinsic length-scale and submicron plasticity in MPEAs. Wang et al. [35] and Zhu et al. [36] have investigated the activation volume and energy for the onset of plastic deformation in two body centered cubic (BCC) HEAs (of compositions NbTaTiZr and NbMoTaTiZr) and a face centered cubic (FCC) HEA (of composition $\mathrm{CoCrFeMnNi)} \mathrm{by} \mathrm{nano-indentation.} \mathrm{They} \mathrm{reported} \mathrm{a} \mathrm{larger} \mathrm{activation} \mathrm{volume} \mathrm{for}$ HEAs compared to conventional metals, attributing it to complex nucleation mechanism. Still, there is limited understanding of the dependence of dislocation nucleation on alloy composition and microstructure, and there are multiple discrepancies in the published studies [2,36].

Here, incipient plasticity and dislocation nucleation were studied using nano-indentation for three different multi-principal element alloys, $\mathrm{CoCrNi}, \mathrm{CoCrFeMnNi}$, and $\mathrm{Al}_{0.1} \mathrm{CoCrFeNi}$. All the three alloys showed a single-phase FCC microstructure without any secondary phases. The three-component CoCrNi alloy showed higher strength than five-component $\mathrm{CoCrFeMnNi}[7,37]$ and was chosen to study the effect of the number of constituents. A comparison was made with a pure metal, $\mathrm{Ni}$, to understand solid solution strengthening effects. In addition, $\mathrm{Al}_{0.1} \mathrm{CoCrFeNi}$ alloy was chosen because it is a model FCC system for comparison with the results of $\mathrm{CoCrFeMnNi}$. The present study paves the way for a fundamental understanding of small-scale deformation mechanisms in multi-principal element alloys as a function of alloy chemistry and microstructure.

\section{Experimental}

Alloys with nominal composition of $\mathrm{CoCrNi}, \mathrm{CoCrFeMnNi}$, and $\mathrm{Al}_{0.1} \mathrm{CoCrFeNi}$ (in mole fractions) were prepared by arc melting the constituent pure elements (purity $>99.99 \%$ ) in Ti-gettered $\mathrm{Ar}$ atmosphere. As-cast alloys were rolled up to a $70 \%$ reduction in thickness. After rolling, they were annealed at $1173 \mathrm{~K}$ for $20 \mathrm{~h}$ to obtain equiaxed dislocation-free grains and to remove residual stress from processing. The annealed samples were polished with $\mathrm{SiC}$ abrasive paper followed by microfiber cloth with $1 \mu \mathrm{m}$ diamond suspension. The polished samples were then put in vibromet with $0.04 \mu \mathrm{m}$ colloidal silica suspension to produce a surface finish equivalent in texture to an electro-polished sample. Crystal structure and phase identification were done using Rigaku III Ultima X-ray diffractometer (XRD Rigaku Corporation, Tokyo, Japan) with $\mathrm{Cu}-\mathrm{K} \alpha$ radiation with wavelength of $1.54 \AA$ A Scanning electron microscopy (SEM) was done using FEI Quanta ESEM (FEI Company, Hillsboro, OR, USA) to analyze the grain size and microstructure of the alloys.

Nano-indentation (Bruker, Minneapolis, MN, USA) was performed using a Berkovich diamond probe. The pop-in behavior of alloys was evaluated with a maximum load of $350 \mu \mathrm{N}$ at $298 \mathrm{~K}, 373 \mathrm{~K}$, and $473 \mathrm{~K}$. One hundred indentations were made at each temperature with $10 \mu \mathrm{m}$ distance between two indents to avoid overlap of their plastic zones. The indentations were done in load control mode with a constant loading rate of $70 \mu \mathrm{N} / \mathrm{s}$. Elevated temperature indentations were performed using XSol600 heating stage with bottom and top heating to avoid any thermal gradients. To prevent oxidation of the sample, a continuous flow of $\mathrm{Ar}+5 \% \mathrm{H}_{2}$ gas mixture was maintained with a flow rate of $1 \mathrm{lit} / \mathrm{min}$.

\section{Results and Discussion}

Figure $1 \mathrm{a}-\mathrm{c}$ show the XRD patterns for the three alloys studied, which confirmed that they formed a simple FCC solid solution and were free of any secondary phases as reported previously [38-40]. The backscattered SEM images of the alloys are shown in Figure 2a-c. All three alloys showed equiaxed microstructure with an average grain size of $\sim 30-60 \mu \mathrm{m}$. They also showed a significant number of annealing twins, which indicates low stacking fault energy in these alloys. 


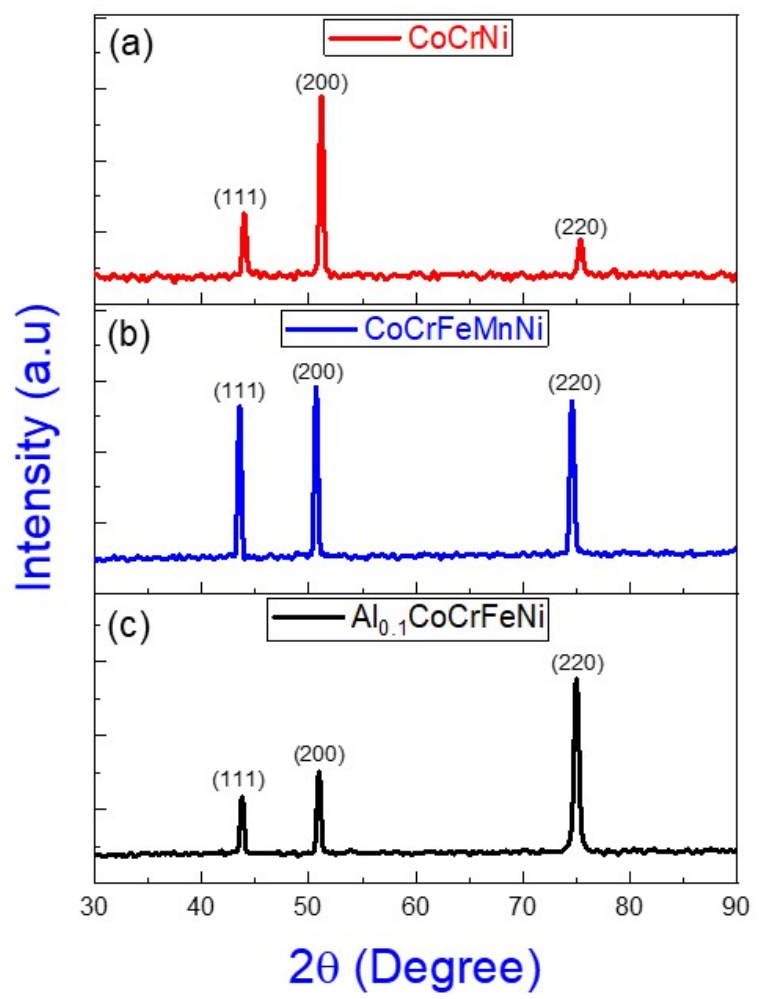

Figure 1. X-ray diffraction (XRD) patterns of multi-principal (a) $\mathrm{CoCrNi}$, (b) $\mathrm{CoCrFeMnNi}$, and (c) $\mathrm{Al}_{0.1} \mathrm{CoCrFeNi}$ alloys.
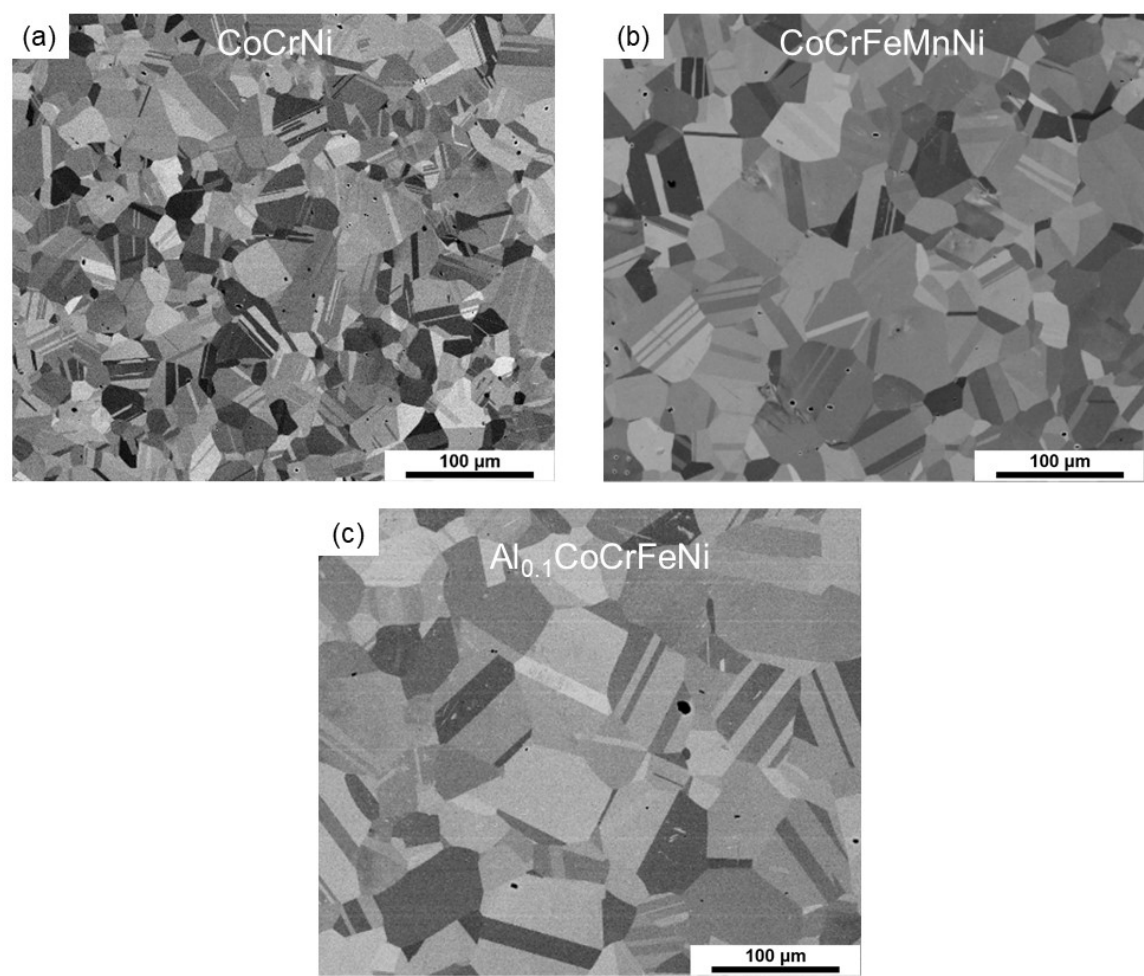

Figure 2. Backscattered scanning electron microscopy (SEM) images of (a) $\mathrm{CoCrNi}$, (b) CoCrFeMnNi, and (c) $\mathrm{Al}_{0.1} \mathrm{CoCrFeNi}$ show equiaxed grains after annealing with grain size $\sim 30-60 \mu \mathrm{m}$. 
Deformation during nano-indentation is initially elastic and follows the Hertzian equation [34].

$$
P=4 / 3 E_{r} R^{\frac{1}{2}} h^{\frac{3}{2}}
$$

where, $P$ is the load applied by the indenter, $R$ is radius of the tip, and $h$ is the indentation depth, $E_{r}$ is the reduced modulus of the indenter-sample combination and is defined by: $\frac{1}{E_{r}}=\frac{1-v^{2}}{E}+\frac{1-v_{i}^{2}}{E_{i}}$. $E, v, E_{i}=1141 \mathrm{GPa}$, and $v_{i}=0.07$ are the Young's modulus and Poisson's ratio of the sample and diamond indenter, respectively. Figure 3 a shows the typical load-displacement curve obtained from nano-indentation along with the Hertzian fit. The reduced modulus of the alloys was obtained from the fitting coefficient of the elastic part and values are summarized in Table 1 and are similar to earlier reports [41,42]. The initial part of the load-displacement curve followed Hertzian contact theory and subsequently deviated due to plastic deformation or elastic-to-plastic transition [34]. The load and displacement at the deviation point was determined for each indent. Figure $3 \mathrm{~b}$ shows the temperature dependence of the pop-in load for the CoCrFeMnNi alloy. The load at first pop-in was found to decrease with increasing temperature; other alloys (CoCrNi and $\mathrm{Al}_{0.1} \mathrm{CoCrFeNi}$ ) and pure $\mathrm{Ni}$ showed a similar trend. This thermal softening has also been reported in several previous studies $[34,36]$.

Table 1. Reduced modulus, activation volume, and activation energy of present face-centered cubic (FCC) alloys.

\begin{tabular}{cccc}
\hline Alloys & $\boldsymbol{E}_{\boldsymbol{r}}(\mathbf{G P a})$ & Activation Volume $\left(\AA^{3}\right)$ & Activation Energy $(\mathbf{e V})$ \\
\hline $\mathrm{Ni}$ & $195 \pm 7$ & 4.12 & $0.23 \pm 0.008$ \\
$\mathrm{CoCrNi}$ & $195 \pm 6$ & 4.69 & $0.27 \pm 0.01$ \\
$\mathrm{CoCrFeMnNi}$ & $180 \pm 4$ & 9.01 & $0.50 \pm 0.007$ \\
$\mathrm{Al}_{0.1}$ CoCrFeNi & $190 \pm 5$ & 8.04 & $0.47 \pm 0.009$ \\
\hline
\end{tabular}
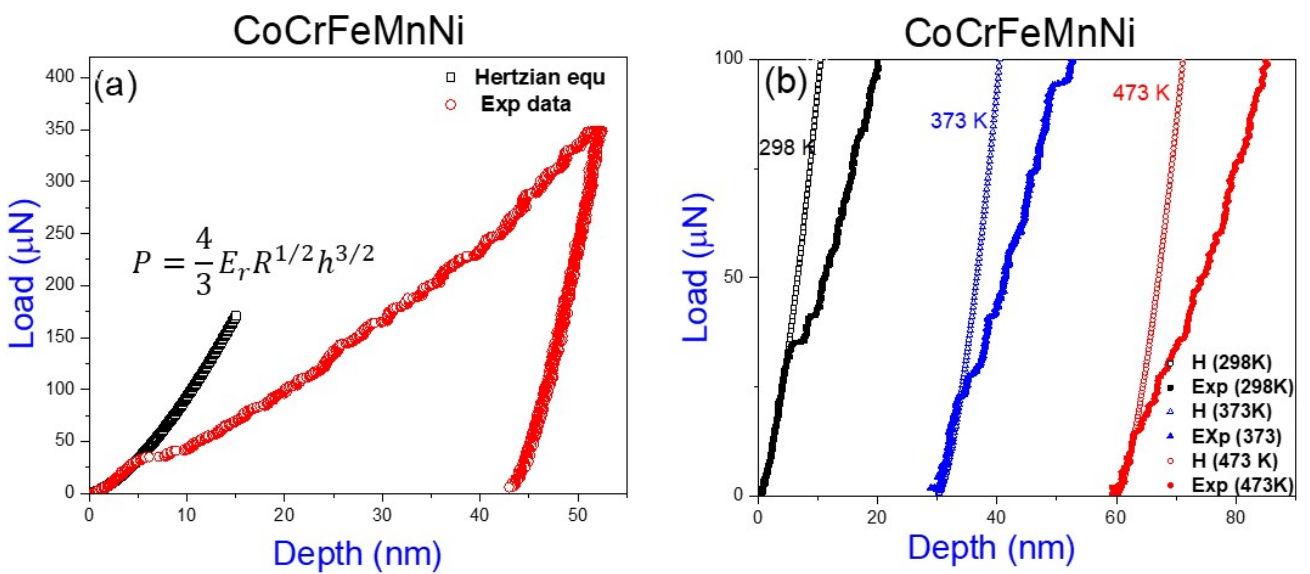

Figure 3. (a) Representative load-displacement curve of $\mathrm{CoCrFeMnNi}$ alloy at room temperature along with the Hertzian; (b) load-displacement curve of $\mathrm{CoCrFeMnNi}$ alloy at different temperatures along with the Hertzian fitting for the elastic section.

Figure 4 shows the statistical distribution of the pop-in load at room temperature ( $298 \mathrm{~K}$ ) and $473 \mathrm{~K}$ for $\mathrm{Ni}$, $\mathrm{CoCrNi}, \mathrm{CoCrFeMnNi}$, and $\mathrm{Al}_{0.1} \mathrm{CoCrFeNi}$. The experimentally obtained data at $373 \mathrm{~K}$ followed a similar trend and has not been included in the figure for clarity. It is also evident from Figure 4 that the pop-in load decreased with increasing temperature. The temperature dependence of displacement burst in multi-principal alloys indicates a stress-biased thermally activated mechanism similar to pure metals [34]. The shear stress beneath the indenter at pop-in is evaluated using Hertzian contact theory as [34]:

$$
p_{m}=\left(\frac{6 P E_{r}^{2}}{\pi^{3} R^{2}}\right)^{1 / 3}
$$




$$
\tau_{m}=0.31 p_{m}
$$

where, $p_{m}$ is the mean contact pressure and $\tau_{m}$ is the maximum shear stress at pop-in, which was in the range of $\sim \frac{\mu}{16}-\frac{\mu}{10}$ (where $\mu$ is the shear modulus) at room temperature for all alloys. As the ideal shear strength of a metal also lies in a similar range $\left(\frac{\mu}{30}-\frac{\mu}{5}\right)$ [36], it may be concluded that the pop-in event involves dislocation nucleation. Using the probabilistic distribution of pop-in stress, an analytical solution has been proposed to evaluate the activation volume and activation energy associated with the displacement burst [34]. Nucleation of dislocation involves an activation barrier which may be overcome by mechanical work done by the indenter or by the combined effect of thermal and mechanical work. The nucleation rate per unit volume, $\dot{n}$ is represented by [34]:

$$
\dot{n}=\eta \exp \left(-\frac{\varepsilon-\sigma V}{k T}\right)
$$

where $\eta$ is a pre-exponential frequency factor, $\epsilon$ is the activation energy barrier for the process, and $\sigma V$ represents the stress bias. In the present condition, the stress bias is the maximum shear stress beneath the indenter. The activation volume of the process may be expressed as [34]:

$$
V=\frac{\pi}{0.47}\left(\frac{3 R}{4 E_{R}}\right)^{2 / 3} k T \alpha
$$

where, $\alpha$ is a time-independent parameter and is obtained from the slope of $\ln [-\ln (1-F)]$ versus $P^{1 / 3}$ plot (as shown in Figure 5), and $F$ is the cumulative probability of the event (as shown in Figure 4). Figure 5 shows the $\ln [-\ln (1-F)]$ versus $P^{1 / 3}$ for $\mathrm{Ni}$ and the three MPEAs at room temperature and $473 \mathrm{~K}$. Using averaged $\alpha$ value obtained from the slope of the curves and Equation (5), the activation volume was calculated. Activation volume for the three alloys and Ni are included in Table 1.
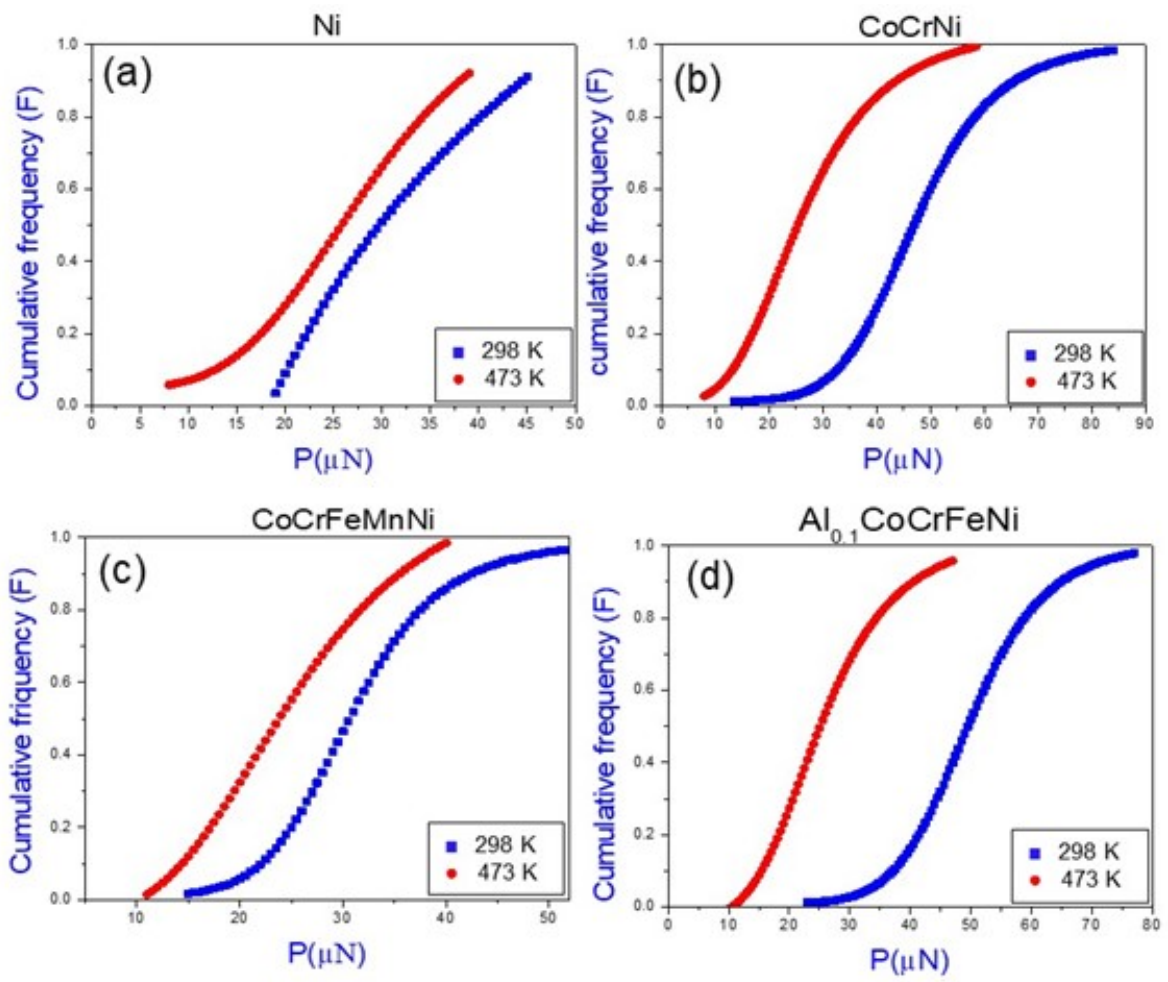

Figure 4. Cumulative fraction of experiment loads at the pop-in events plotted at $298 \mathrm{~K}$ and $473 \mathrm{~K}$ for (a) $\mathrm{Ni}$, (b) CoCrNi, (c) CoCrFeMnNi, and (d) $\mathrm{Al}_{0.1} \mathrm{CoCrFeNi}$ alloys. 

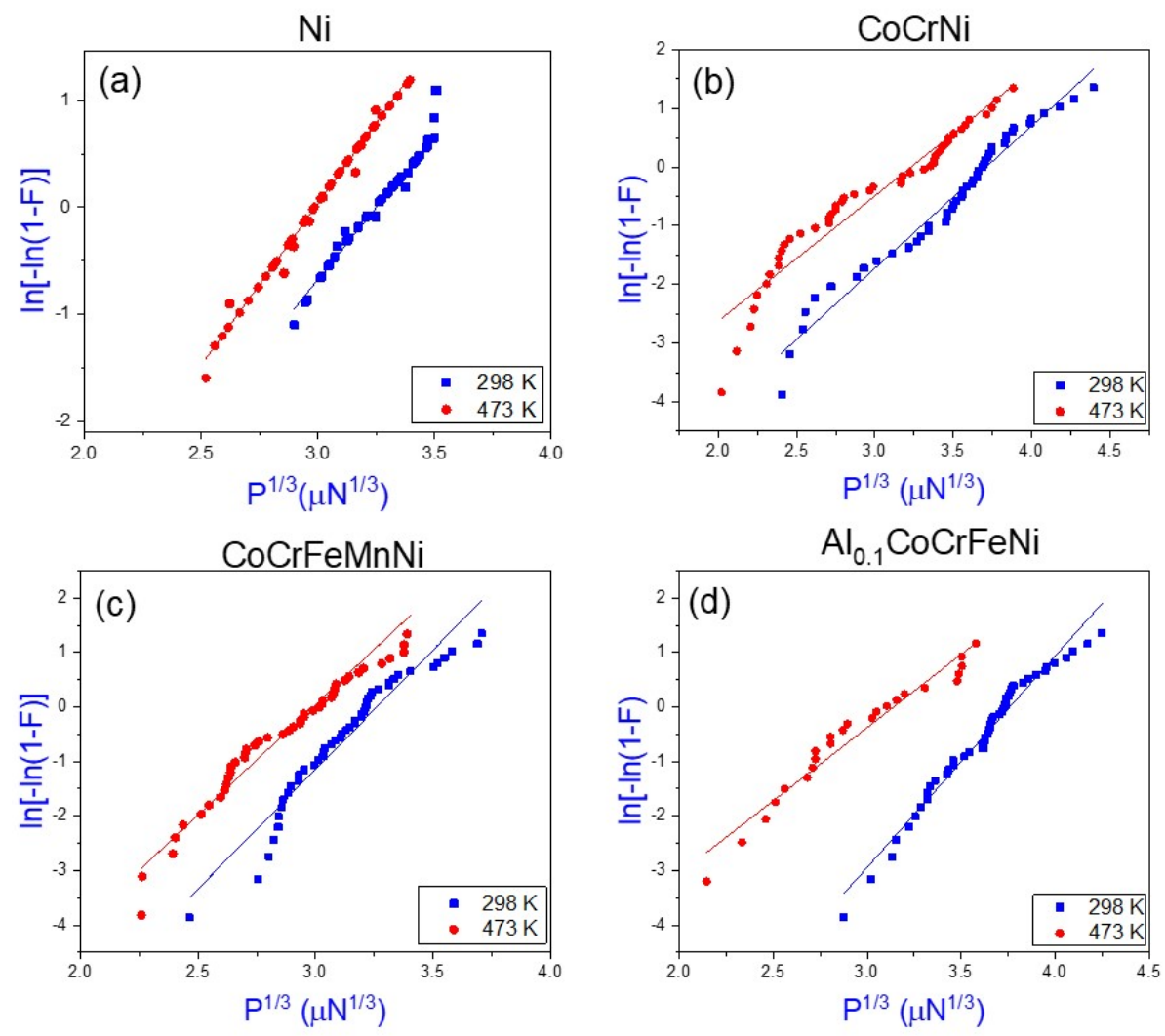

Figure 5. Plot of $\ln [-\ln (1-F)]$ vs. $P^{1 / 3}$ at $298 \mathrm{~K}$ and $473 \mathrm{~K}$ to determine the activation volume from experimental data analysis for (a) $\mathrm{Ni}$, (b) $\mathrm{CoCrNi}$, (c) $\mathrm{CoCrFeMnNi}$, and (d) $\mathrm{Al}_{0.1} \mathrm{CoCrFeNi}$.

Equation (4) indicates that $\epsilon$ also plays an important role in the nucleation process. The activation enthalpy or $\epsilon$ may be evaluated from the pop-in load and activation volume using the equation [34]:

$$
P^{1 / 3}=\gamma k T+\frac{\pi}{0.47}\left(\frac{3 R}{4 E_{R}}\right)^{2 / 3} \frac{\varepsilon}{V}
$$

Figure 6 shows the $P^{1 / 3}$ vs. temperature $(T)$ curves for pure Ni and the MPEAs at three different cumulative frequencies $(F) . P^{1 / 3}$ vs. $T$ shows a linear relation with slope $\gamma k$ and the intercept at $0 \mathrm{~K}$ in $y$-axis representing the second part of Equation (6). With the obtained $P^{1 / 3}$ at $0 \mathrm{~K}$ and the activation volume, $\epsilon$ was evaluated. This represents the energy required for nucleation at $0 \mathrm{~K}$, i.e., all the work done is mechanical work, and there is no thermal work. The activation energy for pure Ni and the MPEAs are summarized in Table 1.

The average activation volume for pop-in in Ni was determined to be $4.12 \AA^{3}$, which falls within a similar range for FCC-Pt [34] and hexagonal close packed (HCP)-Mg [43]. The activation volume and activation energy evaluated for MPEAs using first-order shear bias statistical model were in the range of 4 to $9 \AA^{3}$ and 0.23 to $0.50 \mathrm{eV}$, respectively. These values are much lower than that required for homogenous dislocation nucleation which is higher than $1 \mathrm{eV}$. The discrepancy in activation enthalpy between the experimentally measured value and that expected for homogenous nucleation has been explained by the presence of subcritical loops and thermal fluctuations, while other studies have ruled out these possibilities [44-46]. Vacancy migration during nano-indentation has been proposed as another possible mechanism [2]. But the self-diffusion rate is very slow at room temperature, and vacancy migration energy lies in the range of $1-2 \mathrm{eV}$. The obtained activation enthalpy for the three MPEAs is much lower than the vacancy migration energy. In addition, due to lower diffusivity in equi-atomic multi-component alloys than pure metals, vacancy migration is more difficult in these alloys. Several simulation studies have shown that surface ledges may act 
as potential sites for heterogeneous dislocation nucleation by reducing the activation energy [47,48]. Another proposed mechanism for heterogeneous dislocation nucleation involves hot-spots. At high temperatures, thermal vibrations generate asymmetry which create hot-spot defects below the surface (in the bulk) and act as potential sites for dislocation nucleation, whereas at $0 \mathrm{~K}$ the dislocation nucleates at surface defects $[45,49]$. In the current study, it was observed that the displacement at pop-in decreased with increasing temperature. The average pop-in depth for CoCrFeMnNi alloy corresponding to $298 \mathrm{~K}, 373 \mathrm{~K}$, and $473 \mathrm{~K}$ was $8 \mathrm{~nm}, 5 \mathrm{~nm}$, and $3 \mathrm{~nm}$, respectively. A similar trend was observed for the other two alloys, $\mathrm{CoCrNi}$ and $\mathrm{Al}_{0.1} \mathrm{CoCrFeNi}$. The decreasing trend of pop-in depth with increasing temperature supports the hot-spot mechanism because with increasing temperature the fraction of hot-spot defects increases. This leads to lowering of the depth and load for pop-in. Vacancy clusters and impurity atoms have also been proposed as potential sites for dislocation nucleation [34]. Since the alloys were air cooled after annealing, there is a possibility of having vacancy concentration higher than the equilibrium concentration.
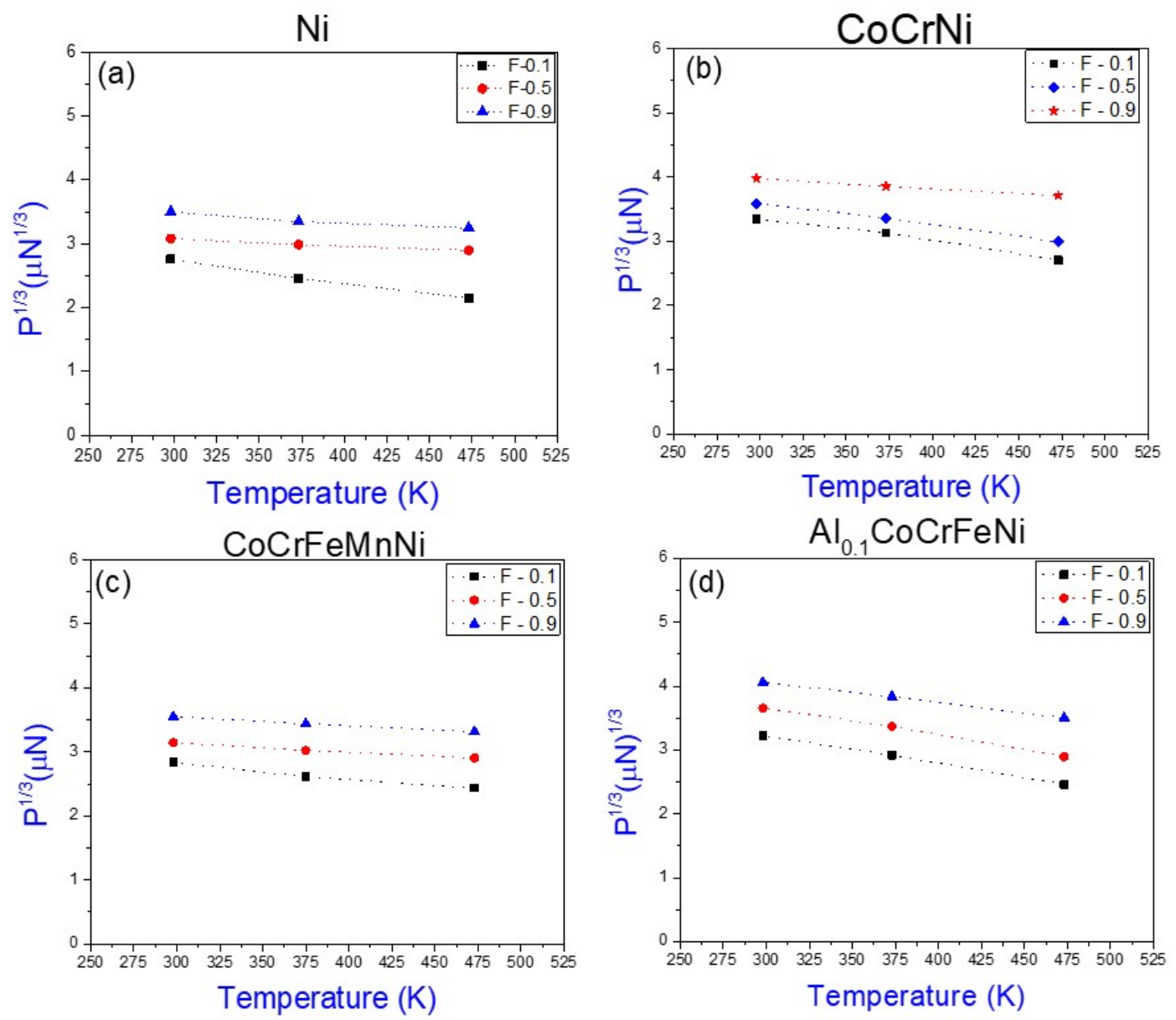

Figure 6. Plot of $P^{1 / 3}$ vs. $T$ to evaluate the activation enthalpy of the first burst for (a) Ni, (b) CoCrNi, (c) $\mathrm{CoCrFeMnNi}$, and (d) $\mathrm{Al}_{0.1} \mathrm{CoCrFeNi}$ alloys.

The activation volume and energy of incipient plasticity for $\mathrm{CoCrFeMnNi}$ and $\mathrm{Al}_{0.1} \mathrm{CoCrFeNi}$ were roughly two times that of pure Ni. This indicates that in the case of MPEAs, the cooperative motion of several atoms is necessary for deformation in comparison with one to one atomic motion in pure metals. The activation volume and energy of the three-component $\mathrm{CoCrNi}$ alloy was marginally higher than pure $\mathrm{Ni}$ (14\% higher) but significantly lower compared to the five-component alloys ( $\sim 50 \%$ lower). This suggests that the dislocation mechanism in this medium entropy alloy (CoCrNi) may be similar to pure metals. The activation volume for dislocation nucleation in BCC TiZNbTa 
and TiZrNbTaMo HEAs were determined to be $\sim 30 \AA^{3}$ [35] which is more than that of the current studied MPEAs. It should also be noted that the obtained activation volume and activation energy for $\mathrm{CoCrFeMnNi}$ in the current work is different compared to that earlier reported by Zhu et al. for the same composition [36]. This may be attributed to the sample preparation conditions and the resulting surface state.

\section{Conclusions}

Shallow depth nano-indentation was performed for multi-principal five-component $\mathrm{Al}_{0.1} \mathrm{CoCrFeNi}$ and $\mathrm{CoCrFeMnNi}$ alloys and three-component $\mathrm{CoCrNi}$ alloy to elucidate the mechanism associated with the onset of plasticity in these alloys. Nano-indentation was performed at three different temperatures of $298 \mathrm{~K}, 373 \mathrm{~K}$, and $473 \mathrm{~K}$. The experimentally obtained data were analyzed within the framework of first-order shear bias statistical model, and the activation volume and activation enthalpy of the process were calculated. The following can be concluded from the obtained experimental and analytical results:

1. The pop-in load marking incipient plasticity was found to decrease with the increase of temperature for each of the three alloys indicating a thermally activated event.

2. At all the temperatures, the pop-in shear stress was not constant but rather occurred over a range of loads. At room temperature, the values of the pop-in shear stress ranged over $\frac{\mu}{16}-\frac{\mu}{10}$ for three alloys.

3. Using a statistical approach, the evaluated activation volume and enthalpy for the five-component multi-principal alloys were around $10 \AA^{3}$ and $0.5 \mathrm{eV}$, respectively. The small values of activation volume and energy suggest heterogeneous dislocation nucleation.

4. The $\mathrm{CoCrFeMnNi}$ and $\mathrm{Al}_{0.1} \mathrm{CoCrFeNi}$ multi-principal alloys showed two times higher activation volume and energy compared to CoCrNi medium entropy alloy and pure Ni suggesting complex cooperative motion of atoms for deformation in these systems.

5. Vacancy migration was ruled out as a possible nucleation mechanism because of the small values of activation volume and energy.

6. Surface defects like ledges, vacancy clusters, and hot-spots created by asymmetry generated due to thermal vibration could be potential sites for heterogeneous dislocation nucleation in these alloys during nano-indentation.

Author Contributions: Conceptualization, S.M. (Sundeep Mukherjee); methodology, S.M. (Sanghita Mridha) and S.M. (Sundeep Mukherjee); validation, S.M. (Sanghita Mridha) and M.S.; formal analysis, M.S.; investigation, S.M. (Sanghita Mridha) and M.S.; resources, S.M. (Sundeep Mukherjee); data curation, S.M. (Sanghita Mridha) and M.S.; writing — original draft preparation, S.M. (Sanghita Mridha) and M.S.; writing-review and editing, M.S. and S.M. (Sundeep Mukherjee); visualization, M.S.; supervision, S.M. (Sundeep Mukherjee); project administration, S.M. (Sundeep Mukherjee).

Funding: This research received no external funding.

Conflicts of Interest: The authors declare no conflict of interest.

\section{References}

1. Yeh, J.W.; Chen, S.K.; Lin, S.J.; Gan, J.Y.; Chin, T.S.; Shun, T.T.; Tsai, C.H.; Chang, S.Y. Nanostructured high-entropy alloys with multiple principal elements: Novel alloy design concepts and outcomes. Adv. Eng. Mater. 2004, 6, 299-303. [CrossRef]

2. Wu, D.; Jang, J.S.C.; Nieh, T.G. Elastic and plastic deformations in a high entropy alloy investigated using a nanoindentation method. Intermetallics 2016, 68, 118-127. [CrossRef]

3. Miracle, D.B.; Senkov, O.N. A critical review of high entropy alloys and related concepts. Acta Mater. 2017, 122, 448-511. [CrossRef]

4. Guo, S.; Ng, C.; Lu, J.; Liu, C.T. Effect of valence electron concentration on stability of fcc or bcc phase in high entropy alloys. J. Appl. Phys. 2011, 109, 103505. [CrossRef] 
5. Zhang, Y.; Zhou, Y.J.; Lin, J.P.; Chen, G.L.; Liaw, P.K. Solid-solution phase formation rules for multi-component alloys. Adv. Eng. Mater. 2008, 10, 534-538. [CrossRef]

6. Zhang, Y.; Zuo, T.T.; Tang, Z.; Gao, M.C.; Dahmen, K.A.; Liaw, P.K.; Lu, Z.P. Microstructures and properties of high-entropy alloys. Prog. Mater. Sci. 2014, 61,1-93. [CrossRef]

7. Gludovatz, B.; Hohenwarter, A.; Thurston, K.V.; Bei, H.; Wu, Z.; George, E.P.; Ritchie, R.O. Exceptional damage-tolerance of a medium-entropy alloy $\mathrm{CrCoNi}$ at cryogenic temperatures. Nat. Commun. 2016, 7, 1-8. [CrossRef] [PubMed]

8. Gali, A.; George, E.P. Tensile properties of high-and medium-entropy alloys. Intermetallics 2013, 39, 74-78. [CrossRef]

9. Schneider, M.; Werner, F.; Langenkämper, D.; Reinhart, C.; Laplanche, G. Effect of Temperature and Texture on Hall-Petch Strengthening by Grain and Annealing Twin Boundaries in the MnFeNi Medium-Entropy Alloy. Metals 2019, 9, 84. [CrossRef]

10. Klimova, M.; Stepanov, N.; Shaysultanov, D.; Chernichenko, R.; Yurchenko, N.; Sanin, V.; Zherebtsov, S. Microstructure and Mechanical Properties Evolution of the Al, C-Containing CoCrFeNiMn-Type High-Entropy Alloy during Cold Rolling. Materials 2018, 11, 53. [CrossRef] [PubMed]

11. Schuh, B.; Pippan, R.; Hohenwarter, A. Tailoring bimodal grain size structures in nanocrystalline compositionally complex alloys to improve ductility. Mater. Sci. Eng. A 2019, 748, 379-385. [CrossRef]

12. Gwalani, B.; Gorsse, S.; Choudhuri, D.; Styles, M.; Zheng, Y.; Mishra, R.S.; Banerjee, R. Modifying transformation pathways in high entropy alloys or complex concentrated alloys via thermo-mechanical processing. Acta Mater. 2018, 153, 169-185. [CrossRef]

13. Gao, M.C. Progress in high-entropy alloys. JOM 2015, 67, 2251-2253. [CrossRef]

14. Kozak, R.; Sologubenko, A.; Steurer, W. Single-phase high-entropy alloys-an overview. Z. Kristallogr.-Cryst. Mater. 2015, 230, 55-68. [CrossRef]

15. Tsai, M.H.; Yeh, J.W. High-entropy alloys: A critical review. Mater. Res. Lett. 2014, 2, 107-123. [CrossRef]

16. Yeh, J.W.; Chen, Y.L.; Lin, S.J.; Chen, S.K. High-entropy alloys-A new era of exploitation. Mater. Sci. Forum 2007, 560, 1-9. [CrossRef]

17. Komarasamy, M.; Kumar, N.; Tang, Z.; Mishra, R.; Liaw, P. Effect of microstructure on the deformation mechanism of friction stir-processed $\mathrm{Al}_{0.1} \mathrm{CoCrFeNi}$ high entropy alloy. Mater. Res. Lett. 2015, 3, 30-34. [CrossRef]

18. Mishra, R.S.; Kumar, N.; Komarasamy, M. Lattice strain framework for plastic deformation in complex concentrated alloys including high entropy alloys. Mater. Sci. Technol. 2015, 31, 1259-1263. [CrossRef]

19. Schuh, C.A.; Lund, A.C. Application of nucleation theory to the rate dependence of incipient plasticity during nanoindentation. J. Mater. Res. 2004, 19, 2152-2158. [CrossRef]

20. Chiu, Y.L.; Ngan, A.H.W. A TEM investigation on indentation plastic zones in $\mathrm{Ni}_{3} \mathrm{Al}(\mathrm{Cr}, \mathrm{B})$ single crystals. Acta Mater. 2002, 50, 2677-2691. [CrossRef]

21. Kiely, J.D.; Houston, J.E. Nanomechanical properties of Au (111), (001), and (110) surfaces. Phys. Rev. B 1998, 57, 12588-12594. [CrossRef]

22. Tymiak, N.I.; Daugela, A.; Wyrobek, T.J.; Warren, O.L. Acoustic emission monitoring of the earliest stages of contact-induced plasticity in sapphire. Acta Mater. 2004, 52, 553-563. [CrossRef]

23. Lorenz, D.; Zeckzer, A.; Hilpert, U.; Grau, P.; Johansen, H.; Leipner, H.S. Pop-in effect as homogeneous nucleation of dislocations during nanoindentation. Phys. Rev. B 2003, 67, 172101. [CrossRef]

24. Syed Asif, S.A.; Pethica, J.B. Nanoindentation creep of single-crystal tungsten and gallium arsenide. Philos. Mag. A 1997, 76, 1105-1118. [CrossRef]

25. Corcoran, S.G.; Colton, R.J.; Lilleodden, E.T.; Gerberich, W.W. Anomalous plastic deformation at surfaces: Nanoindentation of gold single crystals. Phys. Rev. B 1997, 55, R16057. [CrossRef]

26. Bahr, D.F.; Kramer, D.E.; Gerberich, W.W. Non-linear deformation mechanisms during nanoindentation. Acta Mater. 1998, 46, 3605-3617. [CrossRef]

27. Chiu, Y.L.; Ngan, A.H.W. Time-dependent characteristics of incipient plasticity in nanoindentation of a $\mathrm{Ni}_{3} \mathrm{Al}$ single crystal. Acta Mater. 2002, 50, 1599-1611. [CrossRef]

28. Wang, W.; Jiang, C.B.; Lu, K. Deformation behavior of $\mathrm{Ni}_{3} \mathrm{Al}$ single crystals during nanoindentation. Acta Mater. 2003, 51, 6169-6180. [CrossRef]

29. Page, T.F.; Oliver, W.C.; McHargue, C.J. The deformation behavior of ceramic crystals subjected to very low load (nano) indentations. J. Mater. Res. 1992, 7, 450-473. [CrossRef] 
30. Gouldstone, A.; Koh, H.; Zeng, K.Y.; Giannakopoulos, A.E.; Suresh, S. Discrete and continuous deformation during nanoindentation of thin films. Acta Mater. 2000, 48, 2277-2295. [CrossRef]

31. Suresh, S.; Nieh, T.G.; Choi, B.W. Nano-indentation of copper thin films on silicon substrates. Scr. Mater. 1999, 41, 951-957. [CrossRef]

32. Van Vliet, K.J.; Li, J.; Zhu, T.; Yip, S.; Suresh, S. Quantifying the early stages of plasticity through nanoscale experiments and simulations. Phys. Rev. B 2003, 67, 104105. [CrossRef]

33. Gannepalli, A.; Mallapragada, S.K. Atomistic studies of defect nucleation during nanoindentation of Au (001). Phys. Rev. B 2002, 66, 104103. [CrossRef]

34. Mason, J.K.; Lund, A.C.; Schuh, C.A. Determining the activation energy and volume for the onset of plasticity during nanoindentation. Phys. Rev. B 2006, 73, 054102. [CrossRef]

35. Wang, S.P.; Xu, J. Incipient plasticity and activation volume of dislocation nucleation for TiZrNbTaMo high-entropy alloys characterized by nanoindentation. J. Mater. Sci. Technol. 2019, 35, 812-816. [CrossRef]

36. Zhu, C.; Lu, Z.P.; Nieh, T.G. Incipient plasticity and dislocation nucleation of FeCoCrNiMn high-entropy alloy. Acta Mater. 2013, 61, 2993-3001. [CrossRef]

37. Cao, F.; Munroe, P.; Zhou, Z.; Xie, Z. Medium entropy alloy CoCrNi coatings: Enhancing hardness and damage-tolerance through a nanotwinned structuring. Surf. Coat. Technol. 2018, 335, 257-264. [CrossRef]

38. Ahmad, A.S.; Su, Y.; Liu, S.Y.; Stahl, K.; Wu, Y.D.; Hui, X.D.; Ruett, U.; Gutowski, O.; Glazyrin, K.; Liermann, H.P.; et al. Structural stability of high entropy alloys under pressure and temperature. J. Appl. Phys. 2017, 121, 235901. [CrossRef]

39. Sathiyamoorthi, P.; Bae, J.W.; Asghari-Rad, P.; Park, J.M.; Kim, J.G.; Kim, H.S. Effect of annealing on microstructure and tensile behavior of $\mathrm{CoCrNi}$ medium entropy alloy processed by high-pressure torsion. Entropy 2018, 20, 849. [CrossRef]

40. Yang, T.; Tang, Z.; Xie, X.; Carroll, R.; Wang, G.; Wang, Y.; Dahmen, K.; Liaw, P.; Yanwen, Z. Deformation mechanisms of $\mathrm{A}_{10.1} \mathrm{CoCrFeNi}$ at elevated temperature. Mater. Sci. Eng. A 2017, 684, 552-558. [CrossRef]

41. Laplanche, G.; Gadaud, P.; Horst, O.; Otto, F.; Eggeler, G.; George, E.P. Temperature dependencies of the elastic moduli and thermal expansion coefficient of an equiatomic, single-phase CoCrFeMnNi high-entropy alloy. J. Alloys Compd. 2015, 623, 348-353. [CrossRef]

42. Haglund, A.; Koehler, M.; Catoor, D.; George, E.P.; Keppens, V. Polycrystalline elastic moduli of a high-entropy alloy at cryogenic temperatures. Intermetallics 2015, 58, 62-64. [CrossRef]

43. Somekawa, H.; Schuh, C.A. Effect of solid solution elements on nanoindentation hardness, rate dependence, and incipient plasticity in fine grained magnesium alloys. Acta Mater. 2011, 59, 7554-7563. [CrossRef]

44. Sun, Y.Q.; Hazzledine, P.M.; Hirsch, P.B. Cooperative nucleation of shear dislocation loops. Phys. Rev. Lett. 2002, 88, 065503. [CrossRef] [PubMed]

45. Zuo, L.; Ngan, A.H.W.; Zheng, G.P. Size dependence of incipient dislocation plasticity in $\mathrm{Ni}_{3}$ Al. Phys. Rev. Lett. 2005, 94, 095501. [CrossRef] [PubMed]

46. Wo, P.C.; Zuo, L.; Ngan, A.H.W. Time-dependent incipient plasticity in $\mathrm{Ni}_{3} \mathrm{Al}$ as observed in nanoindentation. J. Mater. Res. 2005, 20, 489-495. [CrossRef]

47. Salehinia, I.; Bahr, D.F. The impact of a variety of point defects on the inception of plastic deformation in dislocation-free metals. Scr. Mater. 2012, 66, 339-342.

48. Salehinia, I.; Perez, V.; Bahr, D. Effect of vacancies on incipient plasticity during contact loading. Philos. Mag. 2012, 92, 550-570.

49. Wagner, R.J.; Ma, L.; Tavazza, F.; Levine, L.E. Dislocation nucleation during nanoindentation of aluminum. J. Appl. Phys. 2008, 104, 114311. [CrossRef]

(C) 2019 by the authors. Licensee MDPI, Basel, Switzerland. This article is an open access article distributed under the terms and conditions of the Creative Commons Attribution (CC BY) license (http://creativecommons.org/licenses/by/4.0/). 